\title{
Multivariate Statistical Analysis and Tomographic Processing of Helical Objects
}

\author{
Rasmus R. Schroeder*, Isabel Angert*, Joachim Frank**, and Kenneth C. Holmes* \\ * Max-Planck-Institut for Medical Research, Department of Biophysics, Jahnstr. 29, D-69120 \\ Heidelberg, Germany \\ ** Howard Hughes Medical Institute, Wadsworth Center, Loading Dock J3/P1 South, Albany, New \\ York 12201-0509
}

Helical specimens serve as ideal objects for 3D structure reconstruction from projection images. In principle the helix provides a certain number of projections of the monomeric object for different azimuthal angles along the filament axis. Using Fourier Bessel algorithms it is possible to reconstruct to highest resolution. Problems with this technique are the phase alignment of individual projections, overlapping Bessel functions (which have to be separated by tilted images), and the selection of common helical parameters for objects with an intrinsic helical variability. The use of real space algorithms instead of Fourier based methods for alignment and reconstruction have been presented before [1]. However, the application of common single particle techniques does not exploit the properties of the helical object as an axial tomographic crystal. Here we propose a real space least squares solution to the axial tomographic reconstruction problem of the helical repeat combined with multivariate statistical analysis (MSA) for an enhanced refinement of the azimuthal projection angles. As an example of this procedure we show the reconstruction of the helical actinmyosin complex at a resolution of about $17 \AA$ ( $5 \square \mathrm{FRC}$ criterion).

As in tomography, 3D reconstruction from helical objects combines many projections at different azimuthal angles to arrive at the 3D object. This may be set up as a least squares problem. Since the resulting normal matrix is degenerate, solutions are sought by diagonalising the normal matrix and accepting only the most significant solutions. We have found this a convenient and robust method of reconstruction. It has the advantage of exploiting the perfect local crystal alignment in the helix and does not rely on a global symmetry.

In general, for high resolution reconstructions it is necessary to combine more projection views than are obtainable from one single helical repeat projection. Such independent views are available when images from randomly oriented filaments are combined. Different projections are merged into the initialization of the normal matrix. However, to obtain highest structural resolution a most accurate alignment along the helical axis and in the azimuthal projection angles is necessary. It was found that simple cross correlation techniques of noisy images do not discriminate different azimuthal projection angles of the two-fold actin-myosin complex. In case of the actin-myosin-complex the resulting maximal angle misalignment was on the order of the helical symmetry $\left( \pm 12.7^{\circ}\right)$. This limits resolution to $1-2.5 \mathrm{~nm}$ depending on radius.

The low resolution reconstructed density from such an initial alignment is then used to calculate a set of 2D-projections of the density along different azimuthal angles. Applying MSA these images define a multi-dimensional factor space. Mapping the images onto the strongest factors a 1D-subspace is obtained, which includes the scale for the projection angle. After matching model data to experimental data for signal strength, resolution, and CTF, these experimental data can then directly be mapped into the given factor space. FIG. 1 shows a typical distribution around the sub-space curves. An azimuthal angle can be assigned by determining the smallest distance to the sub-space. In a first refinement of the actin-myosin-complex it was thus possible to reduce the error in azimuthal alignment to less than $\pm 3^{\circ}$. FIG 2 illustrates class averages of experimental data and model images 
classified according to their azimuthal projection angle. The improvement reached in the final density is demonstrated in FIG 3, showing representative slices of the reconstructed density before and after the azimuthal refinement step. The improved object density - particularly at high radius is also illustrated in FIG 4 showing surface representations of the density before and after refinement. At present the density obtained combining 15 defocus series has been refined once. More refinement cycles are under way (see also abstract Schroeder et al., this conference, for new biological results obtained from the new reconstruction).

[1] E.H. Egelmann, Ultramicroscopy 85 (2000) 225
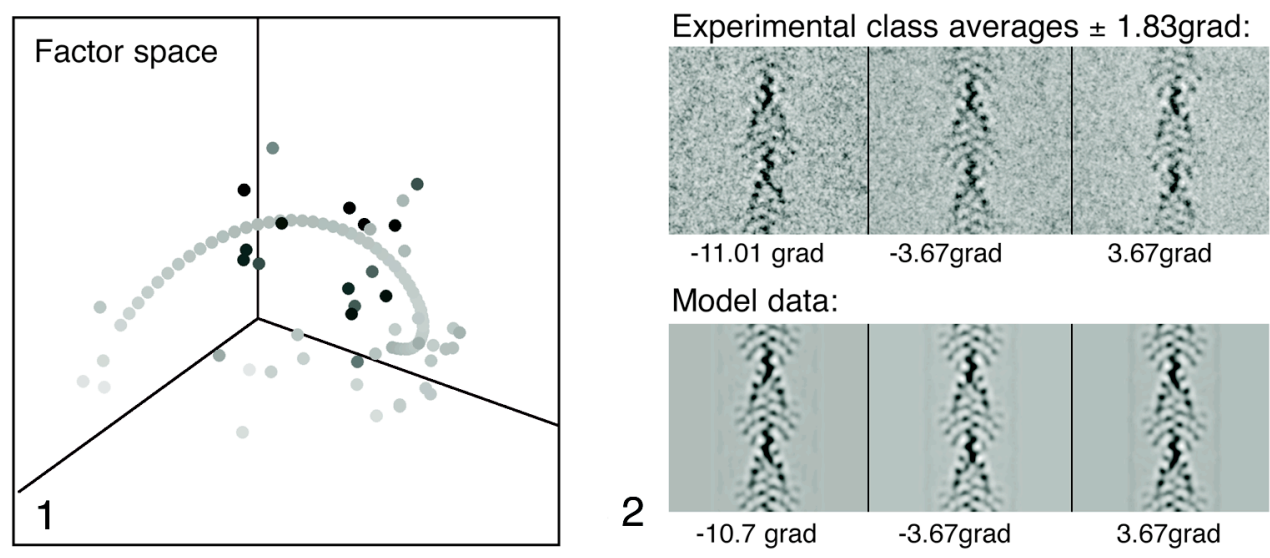

FIG. 13 D representation of the 3 strongest factors after MSA of azimuthal projection model data. The continuous curve represents the 1D sub-space providing the internal scale for the azimuthal angle. The experimental data form the cloud around this sub-space. Azimuthal angles are assigned by the shortest distance of the experimental point to the sub-space.

FIG. 2 Class averages of experimental images after angle assignment and their comparison to the original model images. Note that the resolution of the model data is reduced to more than double the resolution obtained after the refinement step. The angular alignment can therefore be considered "reference free".
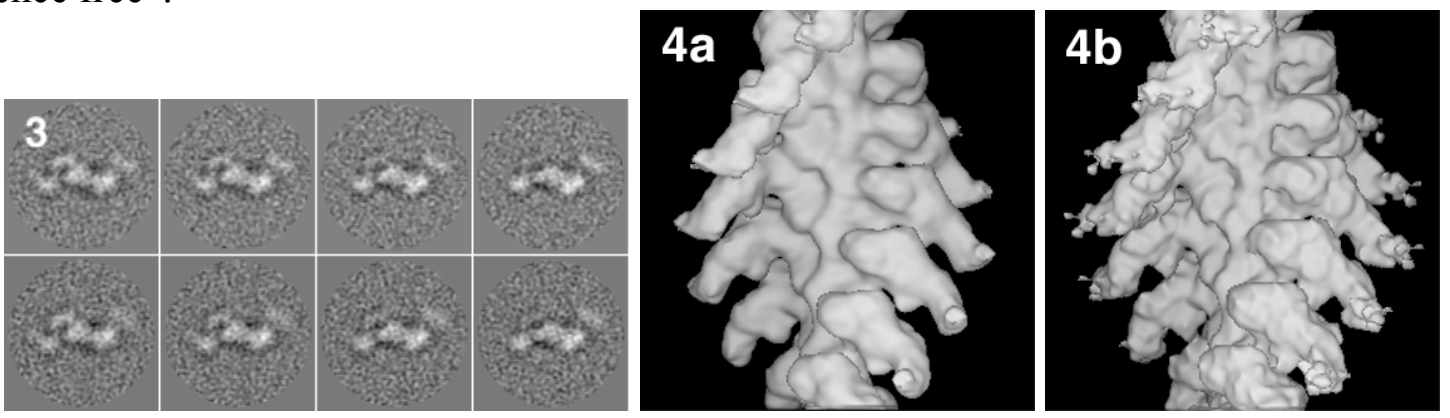

FIG. 3 Comparison of reconstructed slices of the actin-myosin complex after (upper row) and before (lower row) azimuthal refinement. The slices are perpendicular to the filament axis. Note the better definition of the object at higher radius.

FIG. 4 Comparison of reconstructed density of 15 defocus series (about 3000 helical repeats) before (a) and after (b) azimuthal alignment. 